\title{
Hemangioma vertebral cervical con comportamiento agresivo
}

\author{
Cervical vertebral hemangioma with aggressive behavior \\ Jiménez-Ávila JM,* Ibarra-Elizalde VB, ${ }^{\ddagger}$ González-Cisneros AC ${ }^{\S}$
}

Centro Médico Nacional de Occidente, del Instituto Mexicano del Seguro Social. Guadalajara, Jalisco, México.

RESUMEN. Introducción: Los hemangiomas son los tumores primarios más comunes de la columna, principalmente asintomáticos, de hallazgo incidental al realizar un estudio de imagen. La incidencia reportada en autopsias es de $11 \%$ en la columna y lesiones multifocales en 25 a $30 \%$ de los casos. Caso clínico: Hemangioma cervical vertebral con características benignas y estables. Se realizó tratamiento quirúrgico, observando un comportamiento agresivo postquirúrgico dos meses después. Conclusión: El abordaje de la patología vertebral es algorítmico y protocolizado, es necesario determinar el tratamiento con base en la comprensión global de la enfermedad y de acuerdo a las guías de práctica clínica.

Palabras clave: Hemangioma, columna vertebral cervical, tumor benigno.

\section{Introducción}

Los hemangiomas son los tumores primarios más comunes de la columna vertebral, la mayor parte son asintomáticos, por lo general se tratan como un hallazgo incidental al realizar un estudio de imagen. ${ }^{1}$

La incidencia reportada en series de autopsias es de $11 \%$ en la columna vertebral, las lesiones multifocales están presentes en 25 a 30\% de los casos y ocurren más frecuentemente en la columna torácica.
ABSTRACT. Introduction: Hemangiomas are the most common primary tumors of the spine. Mainly asymptomatic Incidental finding when performing an imaging study. The incidence reported at autopsies is $11 \%$ in the spine, multifocal lesions are present in 25 to $30 \%$ of cases. Clinical case: Vertebral cervical hemangioma with benign and stable characteristics that underwent surgical treatment, observing aggressive post-surgical behavior two months later. Conclusion: The approach to vertebral pathology is algorithmic and protocolized, it is necessary to determine the treatment based on the overall understanding of the disease and according to the clinical practice guidelines.

Keywords: Hemangioma, cervical spine, benign tumor.

Los hemangiomas se observan como un trabeculado vertical prominente en las radiografías asemejando un panal de abejas, en la TAC se aprecian como áreas punteadas de esclerosis, lo que se conoce como el signo de «en falda de lunares», el estroma graso y los canales vasculares serpentinosos son los responsables de los componentes observados en las radiografías y la TAC, el sobrecrecimiento graso muestra una señal similar al tejido adiposo subcutáneo y el componente vascular manifiesta una señal de intensidad alta en la difusión ponderada T2 de la RMN.

\footnotetext{
* Cirujano Ortopédico adscrito Clínica de Columna Hospital de Especialidades, de la Unidad Médica de Alta Especialidad, Centro Médico Nacional de Occidente, del Instituto Mexicano del Seguro Social. Guadalajara, Jalisco, México.

‡ Médico residente de Traumatología y Ortopedia, de la Unidad Médica de Alta Especialidad, Centro Médico Nacional de Occidente, del Instituto Mexicano del Seguro Social. Guadalajara, Jalisco, México.

${ }^{\S}$ División ciencias clínicas. TEC de Monterrey campus Guadalajara.
}

Correspondencia:

José María Jiménez-Ávila

Belisario Domínguez Núm. 1000. Col. Independencia, C.P. 44340, Guadalajara, Jal., México.

E-mail: josemajimeneza@hotmail.com

Recibido: 22-04-2020. Aceptado: 15-03-2021.

Citar como: Jiménez-Ávila JM, Ibarra-Elizalde VB, González-Cisneros AC. Hemangioma vertebral cervical con comportamiento agresivo. Acta Ortop Mex. 2021; 35(2): 206-210. https://dx.doi.org/10.35366/101868

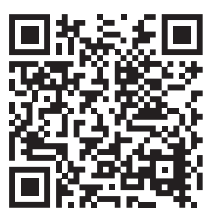


Los hemangiomas son una colección de vasos sanguíneos caracterizados por su delgada pared endotelial, interpuestos entre componentes no vasculares como tejido adiposo, músculo, tejido fibroso o hueso; existen múltiples tipos de hemangiomas descritos (capilar, cavernoso, arteriovenoso y venoso), de los cuales el tipo capilar es el más común.

Los hemangiomas que se extienden hacia los elementos posteriores con compromiso paravertebral y sin gran cantidad de estroma graso intertrabecular son más propensos a desarrollar síntomas.

Las lesiones que contienen menos grasa y más estroma vascular tienden a ser menos comunes y por lo regular están localizadas entre T3 y T9, los hemangiomas que son grandes pueden debilitar y fracturar los cuerpos vertebrales y las fracturas patológicas o la extensión epidural pueden mostrar el signo característico de la «cortina».

Una lesión vertebral que se extiende posteriormente hacia el espacio epidural anterior desplaza el ligamento longitudinal posterior; sin embargo, este desplazamiento se
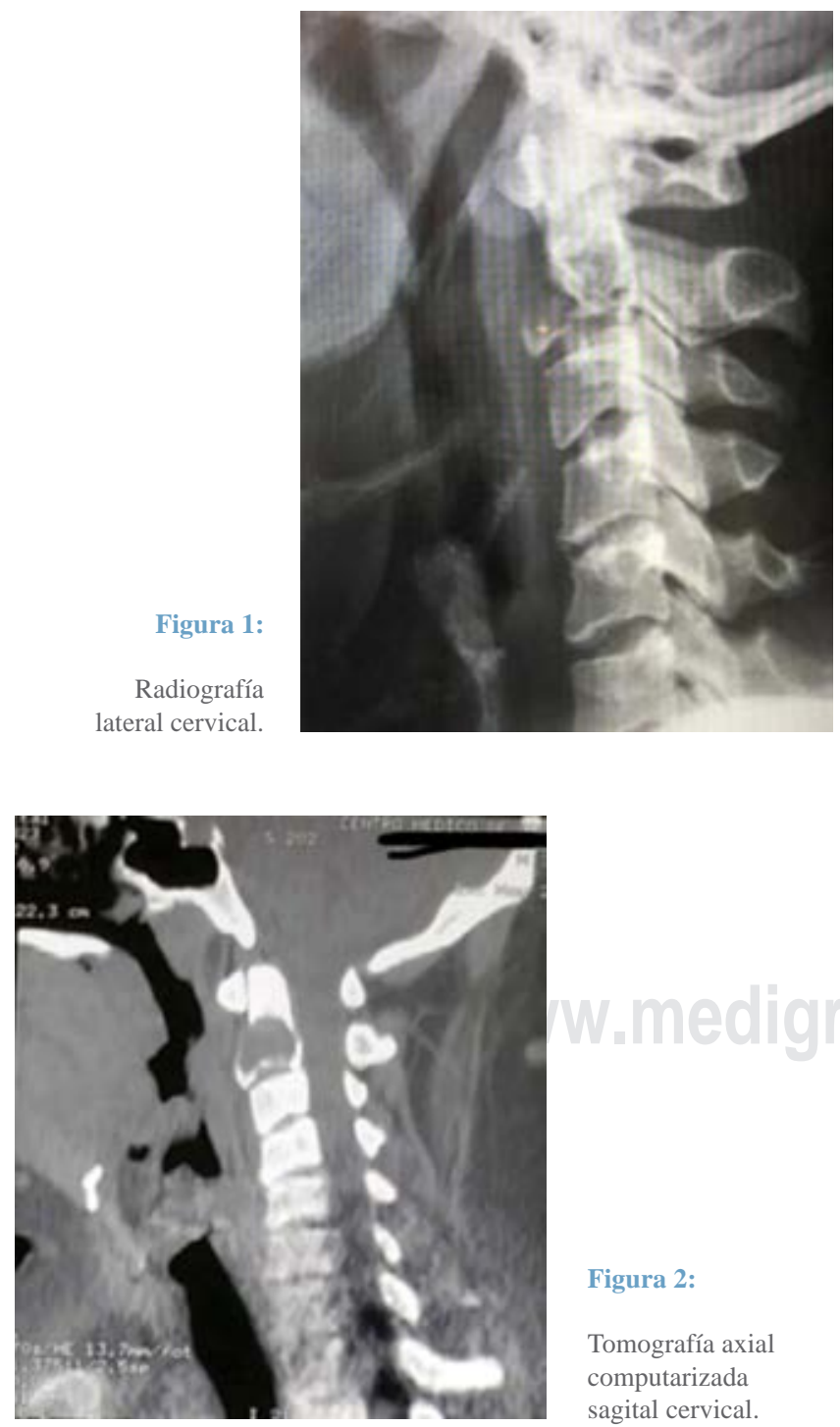

Figura 2:

Tomografía axial computarizada sagital cervical.

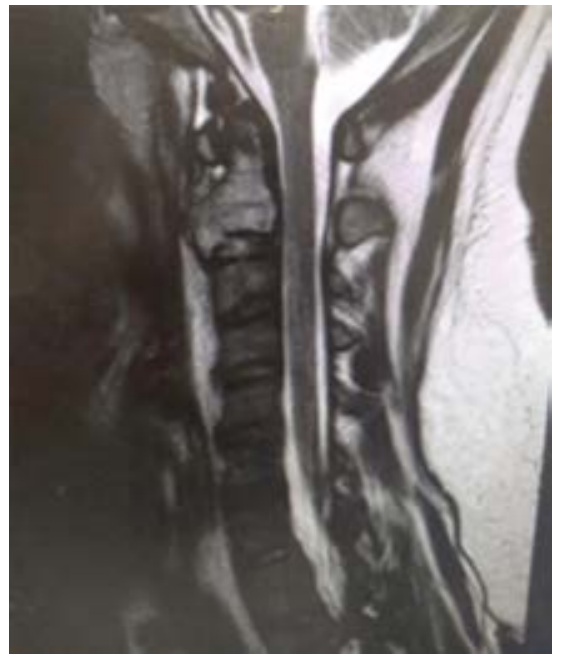

Figura 3:

Resonancia magnética nuclear sagital.

limita medialmente debido a la fuerte fijación medial del ligamento, dando un aspecto bilobulado a la masa epidural cuando se observa en las imágenes axiales, lo que se conoce como el «signo de la cortina». Actualmente, las opciones de tratamiento para los hemangiomas vertebrales sintomáticos incluyen la embolización endovascular, vertebrectomía, escleroterapia y vertebroplastía ${ }^{2}$ y por lo regular tienen un comportamiento benigno que lo único que se requiere es observarlos y valorar que no exista crecimiento anormal que pueda conducir a una compresión neurológica o bien, generar problemas de inestabilidad, por lo cual deberá ser manejado de manera quirúrgica.

\section{Caso clínico}

Paciente masculino de 34 años de edad, es enviado a una unidad de tercer nivel de hospital público para valoración debido a una probable fractura cervical sobre terreno patológico. El evento ocurrió en sedestación cuando ajustaba la inclinación del asiento de su automóvil, súbitamente sufre mecanismo de flexión-extensión cervical ocasionando dolor cervical moderado, previamente había presentado dolor cervical eventual por alrededor de dos meses, no tenía antecedentes personales patológicos y no patológicos significativos.

La exploración neurológica era normal, sin signos y síntomas de compromiso neurológico ni respiratorio; ASIA E, durante su estancia hospitalaria el paciente era capaz de realizar actividades cotidianas sin dificultad como deambular, sentarse, bañarse y alimentarse por sí mismo.

En las radiografías cervicales simples se observó una lesión expansiva, geográfica, circunscrita al cuerpo vertebral de C2; asimismo, se apreció una lesión en T1 con las mismas características de la lesión previamente descrita (Figura 1). En la tomografía axial computarizada (TAC) se observó una lesión hipodensa circunscrita en el cuerpo vertebral de C2, expansiva, que se extendía desde la base del cuerpo vertebral hasta la porción superior de la 
odontoides, además, se detectó una lesión hipointensa, circunscrita, de bordes regulares en el cuerpo vertebral de T1 (Figura 2).

En la resonancia magnética nuclear (RMN) se apreció una lesión hiperintensa en la difusión ponderada en T2, circunscrita, expansiva que se extendía por la totalidad de la vértebra $\mathrm{C} 2$, observando una lesión hipointensa en la difusión ponderada en T1 con las mismas singularidades (Figura 3).

El análisis bioquímico de los marcadores tumorales arrojó como único dato significativo una elevación del antígeno carcinoembrionario (ACE) de $122.5 \mathrm{ng} / \mathrm{ml}$, no se encontraron anormalidades en el resto de los análisis bioquímicos.

Con base en la clasificación de SINS (Spinal Instability Neoplastic Score) se obtuvo una puntuación de seis puntos (localización vertebral -tres puntos-, dolor mecánico o postural -un punto-, características de la lesión -dos puntos-, alineación espinal radiográfica -cero puntos-, involucramiento del cuerpo vertebral -cero puntos- e involucramiento posterior -cero puntos-) catalogándola como una lesión tumoral vertebral estable ( $\leq$ seis puntos) (Tabla 1), considerándola de esta manera y sin compromiso neurológico se le recomendó al paciente la realización de una biopsia por punción transpedicular

Tabla 1: Clasificación de Spinal Instability Neoplastic Score.

Elementos SINS

\begin{tabular}{|c|c|}
\hline Localización & Puntos \\
\hline En la unión (occipital-C2, C7-T2, T11-L1, L5-S1) & 0 \\
\hline Columna móvil (C3-C6, L2-L4) & 2 \\
\hline Semirrígida (T3-T10) & 0 \\
\hline Rígida (S2-S5) & 0 \\
\hline \multicolumn{2}{|l|}{ Alivio del dolor en decúbito o reposo } \\
\hline Sí & 0 \\
\hline No (dolor ocasional pero no mecánico) & 0 \\
\hline Lesión libre de dolor & 0 \\
\hline \multicolumn{2}{|l|}{ Lesión ósea } \\
\hline Lítica & 0 \\
\hline Mixta (lítica/blástica) & 1 \\
\hline Blástica & 0 \\
\hline \multicolumn{2}{|l|}{ Alineación radiográfica de la columna } \\
\hline Subluxación, traslación presente & 0 \\
\hline Deformidad de novo (cifosis, escoliosis) & 0 \\
\hline Alineación anormal & 0 \\
\hline \multicolumn{2}{|l|}{ Colapso del cuerpo vertebral (\%) } \\
\hline$>50$ & 3 \\
\hline$<50$ & 0 \\
\hline $\begin{array}{l}\text { No hay colapso, con más de } 50 \% \\
\text { del cuerpo involucrado }\end{array}$ & 0 \\
\hline Ninguna de las anteriores & 0 \\
\hline \multicolumn{2}{|l|}{ Compromiso de los elementos posterolaterales } \\
\hline Bilateral & 0 \\
\hline Unilateral & 0 \\
\hline Ninguno de los anteriores & 0 \\
\hline Lesión estable (0-6) & $0-6$ \\
\hline Lesión potencialmente inestable (7-12) & $7-12$ \\
\hline Lesión definitivamente inestable (13-18) & $13-18$ \\
\hline Total & 6 \\
\hline
\end{tabular}

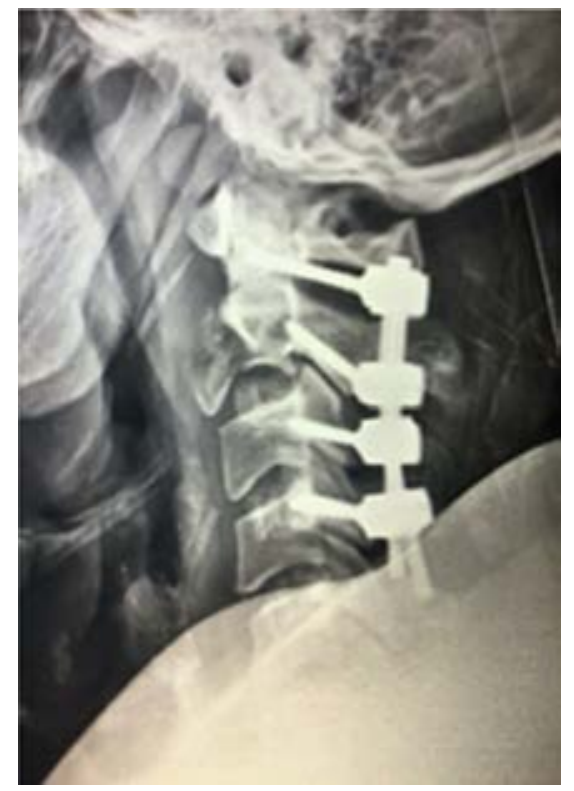

Figura 4:

Radiografía lateral cervical postquirúrgica.

percutánea para determinar nuestra conducta terapéutica; sin embargo, el paciente rechazó esta posibilidad optando por instrumentación posterior cervical con realización de biopsia abierta.

El paciente fue sometido a biopsia transpedicular abierta del cuerpo de C2 y artrodesis cervical mediante instrumentación posterior de C1 a C4 (Figura 4). Como complicación transoperatoria el paciente sufrió lesión de la membrana occipitoatloidea, lo que condicionó una fistula de líquido cefalorraquídeo que requirió reintervención posterior por parte del servicio de neurocirugía, reparada mediante sustituto de duramadre, además desarrolló infección del sitio quirúrgico por $E$. faecalis multirresistente, la cual se resolvió mediante antibioticoterapia. Posteriormente el paciente egresó y se realizó seguimiento en la consulta externa.

El resultado histopatológico de la biopsia reportó un hemangioma intraóseo; sin embargo, tres meses después el paciente reingresa a la unidad hospitalaria por una fractura patológica de T5 asociada a deterioro neurológico (ASIA A).

La tomografía axial computarizada evidenció una lesión ósea en dicho nivel asociada a múltiples lesiones de aspecto «en panal de abejas» diseminadas en la economía vertebral (Figura 5).

La resonancia magnética nuclear vertebral demostró una lesión vertebral tumoral en T5 extracompartimental con afectación medular y paravertebral, con extensión hacia los elementos posteriores vertebrales.

En la región sacra se evidenció una lesión tumoral hiperintensa de bordes regulares, redonda, la cual se alojaba en el cuerpo vertebral de S2 (Figura 6), así como la presencia de múltiples cuerpos vertebrales torácicos y lumbares afectados por lesiones de características similares. Finalmente, tres días después de su reingreso, el paciente fallece a causa de un tromboembolismo pulmonar fulminante. 


\section{Discusión}

Durante cualquier patología vertebral es necesario conocer las directrices que pautan el manejo quirúrgico, es decir, aquellos criterios fundamentales para realizar una cirugía vertebral. Cada paciente debe ser evaluado con base en dos criterios: estabilidad y afectación neurológica. ${ }^{3}$ La estabilidad es definida por White y colaboradores como la capacidad de la columna de mantener relaciones entre las vértebras, en forma tal que no haya daños ni irritación de la médula espinal o de las raíces nerviosas ni deformidad dolorosa. ${ }^{4}$

La clasificación de SINS es un elemento descrito por Fisher y su equipo que permite determinar la estabilidad de una lesión tumoral ${ }^{5}$ y como ya se mencionó previamente, la lesión tumoral que presentaba nuestro paciente se catalogó como estable, mostraba un ángulo de Cobb de la línea de McGregor a C1 de $-27.3^{\circ}$ y un ángulo de Cobb de C2 a C7 de $6.4^{\circ}$, en otras palabras, sin alteraciones en el balance sagital cervical.

Con base en estos hallazgos el paciente era candidato a un tratamiento conservador, en el que realizaría una biopsia percutánea sin estabilizar; sin embargo, el paciente optó por el tratamiento quirúrgico invasivo.

Rex en su algoritmo diagnóstico-terapéutico de tumores primarios de columna indica que el primer paso ante una lesión tumoral solitaria sin déficit neurológico progresivo es la biopsia por punción; si la biopsia determina el diagnóstico es posible establecer una actitud terapéutica; sin

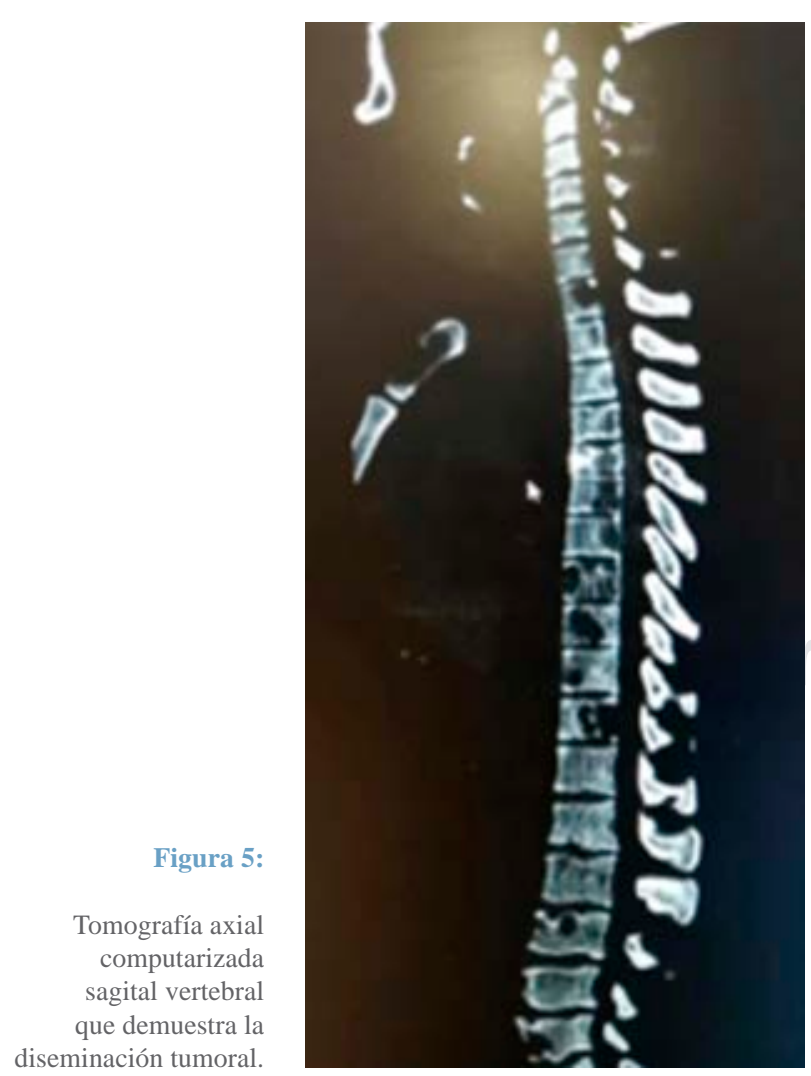

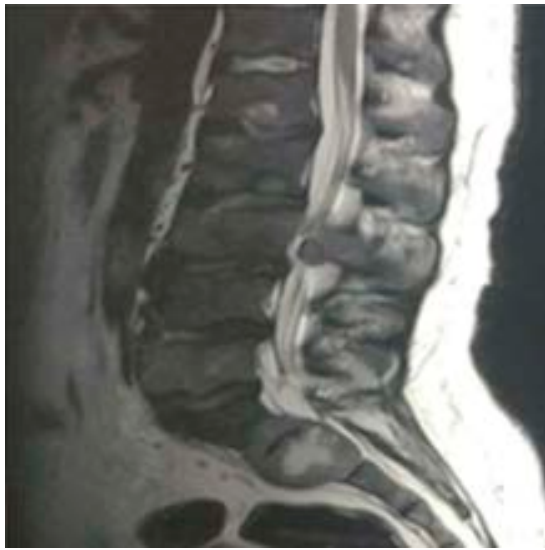

Figura 6:

Resonancia magnética nuclear sagital lumbar donde se observa afectación sacra.

embargo, si la biopsia no aclara el diagnóstico es necesario realizar una biopsia abierta. ${ }^{6}$

En los tumores vertebrales, la biopsia por punción representa el primer paso en el abordaje diagnóstico, por lo que no es posible ofrecer un tratamiento sin un reporte histopatológico previo. La biopsia por punción permite evitar mayor diseminación de células tumorales y por último, permite decidir una terapia definitiva según la histología obtenida.

Benlloch y colaboradores mencionan que los tumores vertebrales pueden metastatizar a través del líquido cefalorraquídeo, el sistema linfático, arterias y venas, aunque los cuerpos vertebrales suelen ser invadidos más frecuentemente a través del plexo venoso paravertebral de Batson. ${ }^{8}$

En el caso clínico en cuestión, el paciente sufrió una fístula de LCR asociada a la manipulación tumoral quirúrgica por la instrumentación posterior vertebral, aunque desconocemos si este hecho propició una diseminación tumoral postquirúrgica a través del LCR o hematógena, ocasionando el reingreso del paciente con múltiples niveles vertebrales afectados y con un comportamiento tumoral agresivo, lo que justifica el papel principal que tiene el abordaje diagnóstico protocolizado de las patologías vertebrales.

\section{Conclusión}

El conocimiento de las directrices que determinan el abordaje de las enfermedades vertebrales es indispensable para el cirujano de columna, por lo tanto es necesario el diagnóstico preciso de las lesiones tumorales vertebrales previo a su tratamiento.

Sólo si las manifestaciones de los pacientes con patología tumoral vertebral representan una afectación de la estabilidad y la función neurológica, está indicado ofrecer un tratamiento quirúrgico especializado.

Ante toda lesión tumoral de columna vertebral solitaria o diseminada es necesario y obligatorio el análisis imagenológico completo de la columna vertebral.

El abordaje de la patología vertebral es algorítmico y protocolizado, por lo que es necesario determinar el tratamiento con base en la comprensión global de la enfermedad y de acuerdo a las guías de práctica clínica. ${ }^{9}$ 


\section{Referencias}

1. Sánchez MJM, Ballesteros MR, Cáceres PE. Infecciones de la columna vertebral. Tumores raquimedulares. En: Delgado MAD. Cirugía ortopédica y traumatología. $4^{\mathrm{a}}$ ed. Madrid: Editorial Médica Panamericana; 2018, 919-930.

2. Ciftdemir M, Kaya M, Selcuk E, Yalniz E. Tumors of the spine. World J Orthop. 2016; 7(2): 109-16.

3. Jiménez-Ávila JM, Sánchez-García O, González-Cisneros AC. Directrices en la decisión del manejo quirúrgico en la cirugía de columna vertebral. Cir Cir. 2019; 87(3): 299-307.

4. White AA, Johnson RM, Panjabi MM, Southwick WO. Biomechanical analysis of clinical stability in the cervical spine. Clin Orthop Relat Res. 1975; (109): 85-96.

5. Fisher CG, DiPaola CP, Ryken TC, Bilsky MH, Shaffrey CI, Berven $\mathrm{SH}$, et al. A novel classification system for spinal instability in neoplastic disease: an evidence-based approach and expert consensus from the spine oncology study group. Spine (Phila Pa 1976). 2010; 35(22): E1221-9.

6. Anderson DG, Vaccaro AR. Decision Making in Spinal Care. 2. ${ }^{\mathrm{a}}$ ed. Nueva York: Thieme Medical Publishers Inc; 2012.

7. Postigo R. Tumores de la columna vertebral. Revista Médica. 2001; 12 (3).

8. Benlloch JM, Morales CA, Bolos TL, Muñoz DS, Valverde BD, Aguirre García R, et al. Metástasis vertebrales. Revista Española de Cirugía Osteoarticular. 2014; 257(49): 17-26.

9. Jiménez-Ávila JM, Rubio-Flores EN, González-Cisneros AC, GuzmánPantoja JE, Gutiérrez-Román EA. Directrices en la aplicación de la guía clínica en la lumbalgia. Cir Cir. 2018; 86(1): 29-37.

Conflicto de intereses: Los autores declaran no tener conflicto de intereses respecto a este trabajo. 